\section{Bosentan for Digital Ulcers in Patients with Systemic Sclerosis: A Prospective 3-year Followup Study}

\section{To the Editor:}

Systemic sclerosis ( $\mathrm{SSc}$ ) is a complex autoimmune connective tissue disease characterized by cutaneous and visceral fibrosis and widespread vascular pathology ${ }^{1}$. Digital ulcers (DU) are a major clinical problem in SSc, occurring in about one-third of patients ${ }^{2}$. DU cause local pain and functional impairment and have a negative effect on quality of life for patients with $\mathrm{SSc}^{2}$. Therapeutic agents potentially used for management of DU include calcium channel blockers, $\alpha$-adrenergic inhibitors, angiotensin II-converting enzyme inhibitors, prostacyclin analogs, phosphodiesterase- 5 inhibitor, and others ${ }^{2}$. Bosentan is a specific orally active dual endothelin receptor antagonist that has been used for the treatment of pulmonary hypertension $(\mathrm{PH})$ and recently for $\mathrm{DU}^{3-5}$. The objective of our study was to examine the effectiveness and safety of bosentan for healing DU in patients with SSc over the long term.

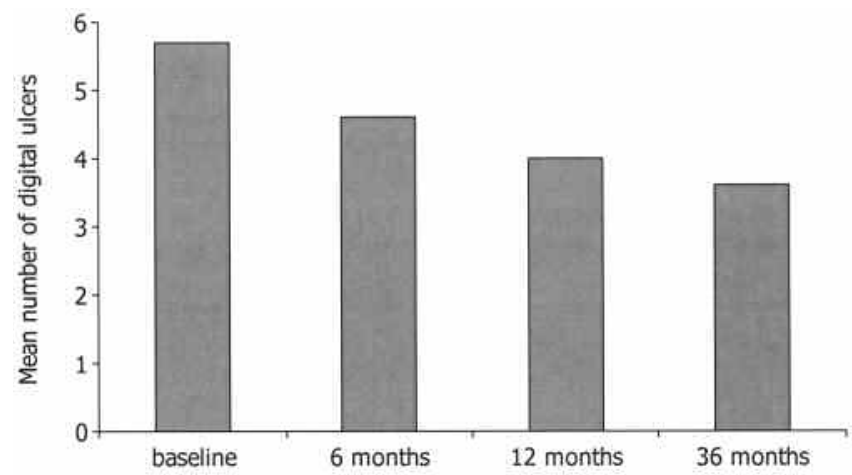

Figure 1. Mean number of digital ulcers (DU) per patient, at baseline and at specific times of followup. Mean number of DU was reduced during followup and at end of study with statistical significance $(p<0.001)$.
For a cohort of 110 patients with SSc, 30 patients with DU were identified. All patients fulfilled the American College of Rheumatology criteria for $\mathrm{SSc}^{6}$ and all were refractory to calcium channel antagonists, angiotensin II inhibitors, or sildenafil.

Patients were treated with bosentan $62.5 \mathrm{mg}$ twice/day for the first month and $125 \mathrm{mg}$ twice/day from the second month for a period of 36 months. The primary endpoints were the number of healed DU and the number of new skin ulcers formed. Pregnant women and nursing mothers and patients with liver or kidney disease, blood dyscrasias, diabetes mellitus, severe cardiopulmonary diseases, or scleroderma renal crisis were excluded from the study.

We gave patients a complete examination and measured the number of treated DU and new ulcers formed. More specifically, all ulcers were measured at entry and every 3 months thereafter for a period of 36 months. Skin biopsies of affected areas were performed in 5 patients, at the same place before and after therapy. From these 30 patients, 4 were excluded: 2 had abnormal liver function test, 1 had thrombocytopenia, and 1 had diabetes mellitus with liver steatosis. Thus, 26 patients were investigated. There were 22 women and 4 men with mean age of $60.0 \pm 6.5$ years and mean disease duration of $12.5 \pm 8.6$ years. Sixteen had limited scleroderma, while 10 had the diffuse type of the disease. Five patients had PH and 3 of them also had pulmonary fibrosis. Twenty were treated with methotrexate ( $0.2 \mathrm{mg} / \mathrm{kg}$ body weight) plus small doses of prednisone ( $<7.5 \mathrm{mg} /$ day $), 3$ with mycophenolate mofetil and small doses of steroids, and 4 with prednisone $(<7.5 \mathrm{mg} / \mathrm{day})$.

The mean number of DU per patient was reduced during the followup (6th mo and 12th mo) and at the end of the study (36th mo). The change was statistically significant $(\mathrm{p}<0.001)$ for the 3 observation times (Figure 1). Healing of skin ulcers occurred in $17 / 26$ patients $(65 \%)$ after a median period of 25 weeks (range 8-26 wks). A clear improvement was noted in 4 patients (15\%) and new ulcers developed in $5(19 \%)$. Of interest is the fact that patients with PH had clinical improvement (by measuring 6 min walk time) and 3 of them had healed DU, while 2 presented clear improvement. Skin biopsy from the affected area showed a significant histological improvement (Figure 2A, 2B). Three patients discontinued the study
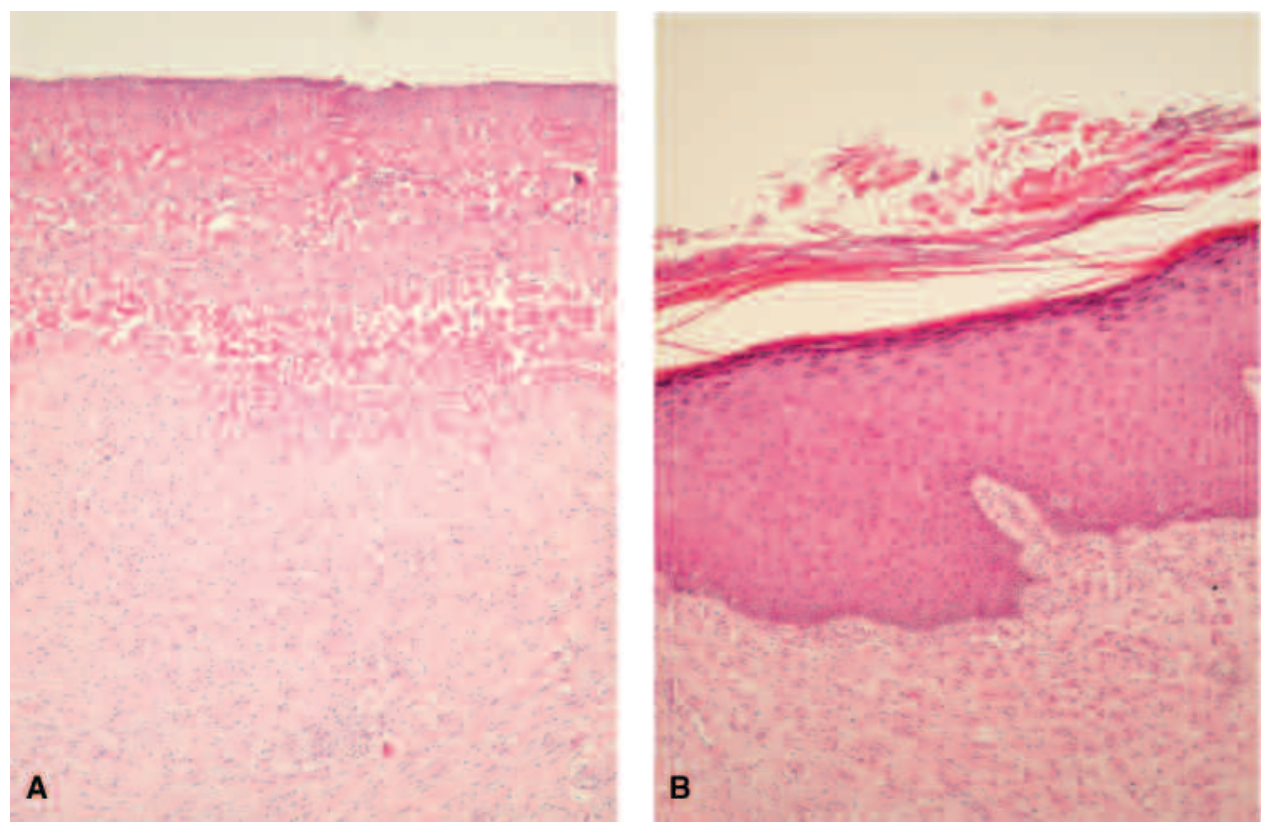

Figure 2. Skin biopsy before and after treatment with bosentan. (A) Before: the epidermis is ulcerated and surface coated by fibrinoid material with necrotic debris. The papillary dermis shows a dense infiltrate of neutrophils extending throughout the sclerosing reticular dermis. (B) After: the epidermis shows orthokeratosis, hypergranulosis, and extensive acanthosis. The dermis is sclerotic with increased number of fibrocytes. It shows dilated capillaries and dense infiltrate of lymphocytes, plasma cells, and occasional histiocytes. 
(11.5\%): 2 because they had elevated transaminase levels after 1 year of treatment, while 1 patient had reduction of hematocrit after 16 months of therapy.

DU are a common and debilitating problem for patients with SSc. Several agents are currently being used for the treatment and prevention of $\mathrm{DU}^{2,7-9}$. Recent double-blind studies, where the use of bosentan was compared to placebo, showed a decrease in the rate of new ulcer formation and improvement of the overall hand function score ${ }^{5}$. Other small series of patients showed the beneficial effects of bosentan on treating DU in $\mathrm{SSc}^{7}$. A recent open-label study of 15 patients with SSc and DU using bosentan for a median period of 24 months revealed that bosentan was safe and effective in these patients ${ }^{10}$. Ours is the longest prospective study that has evaluated the number of healed DU and new ulcer formation. We observed that healed ulcers occurred in $17(65 \%)$ patients and a clear improvement in 4 more patients.

A limitation of our study is the small number of patients and its open-label design without a control group. We should emphasize, however, that all our patients were refractory to previous treatments and this is the only study in which DU improvement was demonstrated by skin biopsy.

Treatment with bosentan in patients with SSc has an acceptable toxicity profile and is effective in preventing and treating DU in a longterm fashion. However, other controlled studies with larger numbers of patients are needed to demonstrate the effectiveness and safety of bosentan in patients with SSc.

NIKI TSIFETAKI, MD, Registrar of Rheumatology; VASSILIOS BOTZORIS, MD, Fellow in Rheumatology, Rheumatology Clinic, Department of Internal Medicine, Medical School, University of Ioannina, Ioannina; YANNIS ALAMANOS, MD, Associate Professor of Hygiene and Epidemiology, Department of Public Health, Medical School, University of Patras, Patras; EVAGGELIA ARGYRIOU, MD, Fellow in Rheumatology, Rheumatology Clinic, Department of Internal Medicine; AIKATERINI ZIOGA, MD, Registrar of Pathology, Department of Pathology; ALEXANDROS A. DROSOS, MD, FACR, Professor of Medicine/Rheumatology, Head, Rheumatology Clinic, Department of Internal Medicine, Medical School, University of Ioannina, 45110 Ioannina, Greece. Address reprint requests to Prof. Drosos.

E-mail: adrosos@cc.uoi.gr

\section{REFERENCES}

1. Charles C, Clements P, Furst DE. Systemic sclerosis: hypothesis-driven treatment strategies. Lancet 2006;367:1683-91.

2. Denton C, Korn J. Digital ulceration and critical digital ischemia in scleroderma. Scleroderma Care Res 2003;1:12-6.

3. Roux S, Breu V, Ertel SI, Clozel M. Endothelin antagonism with bosentan: a review of potential applications. J Mol Med 1999;77:364-76.

4. Rubin LJ, Badesch DB. Evaluation and management of the patient with pulmonary arterial hypertension. Ann Intern Med 2005;143:282-92.

5. Korn JH, Mayes M, Matucci Cerinic M, et al. Digital ulcers in systemic sclerosis: prevention by treatment with bosentan, an oral endothelin receptor antagonist. Arthritis Rheum 2004;50:3985-93.

6. Preliminary criteria for the classification of systemic sclerosis (scleroderma). Subcommittee for scleroderma criteria of the American Rheumatism Association Diagnostic and Therapeutic Criteria Committee. Arthritis Rheum 1980;23:581-90.

7. Chung L, Fiorentino D. Digital ulcers in patients with systemic sclerosis. Autoimmun Rev 2006;5:125-8.

8. Abou Raya A, Abou-Raya S, Helmii M. Statins: potentially useful in therapy of systemic sclerosis-related Raynaud's phenomenon and digital ulcers. J Rheumatol 2008;35:1801-8.

9. Kawald A, Burmester GR, Huscher D, Sunderkotter C, Riemekasten G. Low versus high-dose iloprost therapy over 21 days in patients with secondary Raynaud's phenomenon and systemic sclerosis: a randomized, open, single-center study. J Rheumatol 2008;35:1830-7.

10. García de la Peña-Lefebvre P, Rodríguez Rubio S, Valero Expósito $\mathrm{M}$, et al. Long-term experience of bosentan for treating ulcers and healed ulcers in systemic sclerosis patients. Rheumatology 2008;47:464-6.

J Rheumatol 2009;36:7; doi.10.3899/jrheum.080992 